\title{
Impaired self-other differentiation in frontotemporal dementia due to the C9ORF72 expansion
}

\author{
Laura E Downey, Colin J Mahoney, Martin N Rossor, Sebastian J Crutch and Jason D Warren*
}

\begin{abstract}
Introduction: An expanded hexanucleotide repeat in the C9ORF72 gene has recently been identified as an important cause of frontotemporal dementia and motor neuron disease; however, the phenotypic spectrum of this entity and its pathophysiologic basis have yet to be fully defined. Psychiatric features may be early and prominent, although a putative cortico-thalamo-cerebellar network has been implicated in the pathogenesis of the clinical phenotype. Differentiation of self from others is a core cognitive operation that could potentially link network disintegration with neuropsychiatric symptoms in C9ORF72-associated frontotemporal dementia.

Methods: We undertook a detailed behavioral analysis of self-other attribution in a 67-year-old male patient with behavioral variant frontotemporal dementia (bvFTD) due to the C9ORF72 expansion by using a novel paradigm requiring differentiation of the effects of self- and non-self-generated actions. The patient's performance was assessed in relation to two older male patients with bvFTD not attributable to the C9ORF72 expansion and four healthy older male subjects.

Results: Compared with the healthy control group, the patient with the C9OFR72 mutation showed a deficit of self-other differentiation that was disproportionate to his otherwise relatively indolent clinical phenotype. The performance of the other patients with bvFTD was similar to that of healthy subjects.

Conclusion: We propose that impaired self-other differentiation is a candidate mechanism for neuropsychiatric decline in association with the C9ORF72 expansion. We offer this preliminary observation as a stimulus to further work.
\end{abstract}

\section{Introduction}

An expanded hexanucleotide repeat in the C9ORF72 gene has recently been identified as an important cause of frontotemporal dementia and motor neuron disease [1-3]; however, the phenotypic spectrum of this entity and its pathophysiologic basis have yet to be fully defined. Psychiatric manifestations including delusions, hallucinations, and severe anxiety disorders have been identified as frequent and prominent and may be presenting features $[1,3,4]$. Although detailed neuroanatomic-phenotypic correlation has yet to be undertaken in the C9ORF72 mutation spectrum, a culprit cortico-thalamo-cerebellar network has been identified as a potential substrate for

\footnotetext{
* Correspondence: jason.warren@ucl.ac.uk

* Correspondence: jason.warren@ucl.ac.uk London, London, UK
} London, London, UK

\section{()

certain clinical features, in particular, for neuropsychiatric symptoms [1]. Differentiation of self from others is a core cognitive operation that could potentially link network disintegration with neuropsychiatric symptoms in C9ORF72associated frontotemporal dementia. Disordered self-other differentiation has been implicated in the pathogenesis of various psychiatric conditions, including schizophrenia, out-of-body autoscopic experiences, anxiety, and depression [5-8]. Neuroimaging evidence in the healthy brain suggests that the cerebellum is a key component of distributed cortico-subcortical circuitry that represents and calibrates the effects of actions generated by oneself and others [5,9].

We recently had the opportunity to investigate this issue in a patient, NT, with the C9ORF72 expansion, who exhibited an indolent phenotype of behavioral variant frontotemporal dementia (bvFTD) with relative preservation of 
many cognitive abilities. We designed a novel behavioral paradigm specifically to assess NT's ability to distinguish between the effects of self- and non-self-generated actions. We assessed NT in relation to a group of healthy older male control subjects and two older male patients (DC1 and DC2) with bvFTD not attributable to the $c 9 O R F 72$ expansion.

\section{Methods \\ Case descriptions \\ NT}

NT is a 67-year-old right-handed male retired information technology consultant with a postgraduate degree qualification. He presented with an insidious decline in personality and behavior extending over some 20 years. His increasing impassivity, impulsivity, and distractibility had led to his retirement from work. His wife reported that he was less gregarious and less empathic, more obsessional about money, and showed an increased preference for sweet foods. He made tactless remarks to strangers and had only limited insight into his difficulties. No concern had been expressed about his episodic memory or route-finding abilities, and his language, though somewhat tangential, remained articulate. No history suggested delusions, hallucinations, or other perceptual disturbance. A family history existed of behavioral decline in NT's mother from age 50, and dementia with parkinsonism in a maternal uncle. NT's general neurologic examination was unremarkable; in particular, no features of motor neuron disease were found. Longitudinal neuropsychological assessment over a 9-year interval demonstrated a largely stable profile with superior performance in most cognitive domains and only a mild relative weakness of aspects of executive function; NT's general neuropsychological data at the time of the experimental assessment are summarized in Table 1. NT fulfilled current consensus criteria for a syndromic diagnosis of bvFTD [10].

Serial registered $T_{1}$-weighted volumetric brain MRI demonstrated progressive, diffuse cerebral and cerebellar volume loss; brain MRI at the time of the behavioral assessment (Figure 1) showed mild atrophy, predominantly affecting left frontal and perisylvian regions. Peripheral electrophysiological studies revealed no evidence of anterior horn cell dysfunction or peripheral neuropathy. Genomic screening identified a pathologic hexanucleotide expansion in the C9ORF72 gene.

\section{DC1}

DC1 is a 63-year-old right-handed male retired plumber. He presented with a 6-year history of progressive personality change, initially with uncharacteristic aggressive outbursts and supervening social withdrawal, obsessionality, behavioral rituals, and sweet tooth. Memory impairment was another prominent early feature. No history of
Table 1 Demographic and neuropsychological characteristics of patients and healthy controls

\begin{tabular}{|c|c|c|c|c|}
\hline & NT & DC1 & $\mathrm{DC} 2$ & $\begin{array}{l}\text { Healthy } \\
\text { controls } \\
(n=4)\end{array}$ \\
\hline Age (years) & 67 & 63 & 74 & $67(6.03)$ \\
\hline Education (years) & 15 & 12 & 16 & $15(2)$ \\
\hline \multicolumn{5}{|l|}{ General intellect } \\
\hline WASI VIQ & 118 & 63 & 115 & $72(3.4)$ \\
\hline WASI PIQ & 132 & 91 & 111 & $57(2.6)$ \\
\hline NART (/50) & $>75$ th & $<25$ th & >90th & $41(4.8)$ \\
\hline \multicolumn{5}{|l|}{ Episodic memory } \\
\hline RMT Words (/50) & $\begin{array}{l}25- \\
50 \text { th }\end{array}$ & $<5$ th & 5-10th & $44(5.2)$ \\
\hline RMT Faces (/50) & $>95$ th & $<5$ th & $<5$ th & $46(6.1)$ \\
\hline \multicolumn{5}{|l|}{ Executive function } \\
\hline D-KEFS Stroop word & $>50$ th & $>50$ th & $>50$ th & $21(3.5)$ \\
\hline $\begin{array}{l}\text { D-KEFS Stroop } \\
\text { Inhibition }\end{array}$ & $>50$ th & $>50$ th & $>50$ th & $53(15.5)$ \\
\hline \multicolumn{5}{|l|}{ Semantic processing } \\
\hline BPVS (/150) & 146 & 103 & 138 & $149(1.5)$ \\
\hline \multicolumn{5}{|l|}{ Other skills } \\
\hline GNT (/30) & $>50$ th & $<1 \mathrm{st}$ & $<1 \mathrm{st}$ & $27(2.9)$ \\
\hline Forward DS (/12) & $>95$ th & $\begin{array}{l}\text { 75th- } \\
90 \text { th }\end{array}$ & $\begin{array}{l}\text { 90th- } \\
95 \text { th }\end{array}$ & $8(1)$ \\
\hline Reverse DS (/12) & $>50$ th & $\begin{array}{l}\text { 50th- } \\
75 \text { th }\end{array}$ & $\begin{array}{l}\text { 90th- } \\
95 \text { th }\end{array}$ & $6(1)$ \\
\hline Addition & $>95$ th & $>50$ th & 25-50th & $7(1.2)$ \\
\hline Subtraction & $>95$ th & $\begin{array}{l}\text { 25th- } \\
50 \text { th }\end{array}$ & $>50$ th & $8(3.3)$ \\
\hline VOSP & $>95$ th & 25-50th & $>50$ th & $19(0.5)$ \\
\hline $\mathrm{CBI}$ & 52 & 203 & 46 & $\mathrm{n} / \mathrm{a}$ \\
\hline \multicolumn{5}{|l|}{$\begin{array}{l}\text { Experimental } \\
\text { conditions }^{b}\end{array}$} \\
\hline Self $(/ 10)$ & 10 & 10 & 10 & $9.5(9-10)$ \\
\hline Synchronous (/10) & 3 & 7 & 5 & $6.5(5-7)$ \\
\hline Asynchronous (/10) & 4 & 9 & 10 & $9.5(9-10)$ \\
\hline
\end{tabular}

Percentile ranking scores of neuropsychological performance are shown. Raw scores are listed where percentile scores are not available or applicable. BPVS, British Picture Vocabulary Scale; $\mathrm{CBI}$, Cambridge Behavioural Inventory total score (across domains including mood, beliefs, challenging behaviors, disinhibition, eating, sleep, stereotypies, motivation, and insight (Wedderbum et al., 2008)); D-KEFS Stroop (word and inhibition), Delis-Kaplan Executive Function System; DS, digit span; GNT, Graded Naming Test; n/a, not applicable; NART, National Adult Reading Test; RMT, Recognition Memory Test; Verbal Intelligence score; VOSP, Visual Object and Space Perception battery; WASI PIQ, Wechsler Abbreviated Scale of Intelligence-Performance Intelligence score; WASI VIQ, Wechsler Abbreviated Scale of Intelligence, ${ }^{a}$ Mean (standard deviation) values shown, except for experimental conditions, in which mean (range) values shown. ${ }^{b}$ See text for explanation.

delusions, hallucinations, or other perceptual disturbance was present. The patient had a known family history of early-onset frontotemporal dementia with autosomal dominant inheritance. His general neurologic examination was normal. In particular, no features of motor neuron disease were noted. Serial neuropsychological assessments over a 6-year interval demonstrated progressive impairment, particularly affecting naming and 


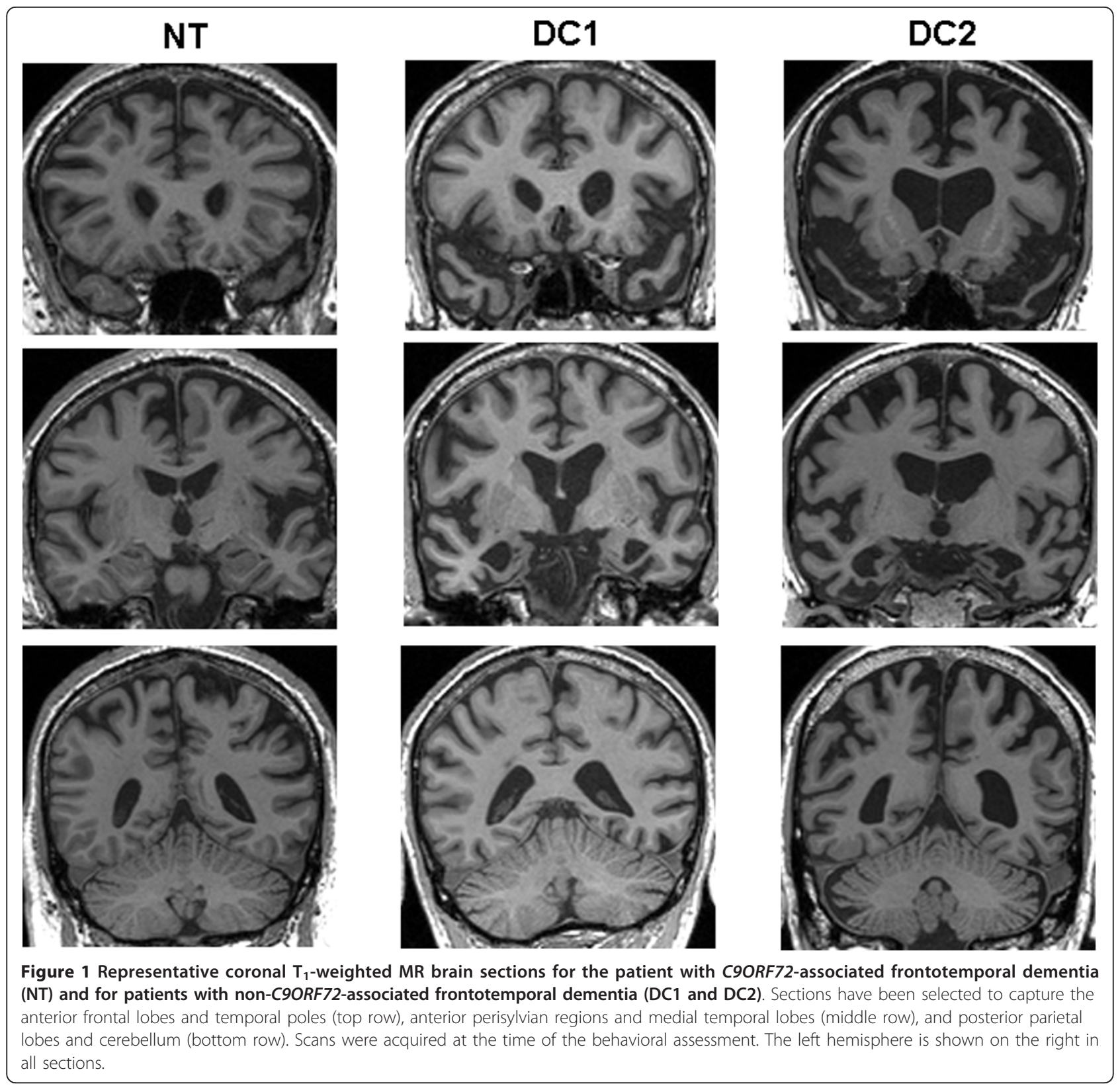

executive functions; DC1's general neuropsychological data at the time of the experimental assessment are summarized in Table 1. DC1 fulfilled current consensus criteria for a syndromic diagnosis of bvFTD [10].

Serial registered $T_{1}$-weighted volumetric brain MRI demonstrated progressive cerebral atrophy, most marked in the anteromesial temporal lobes, which were relatively symmetrically affected (Figure 1). Genomic screening demonstrated a c.1216C $>\mathrm{T}$ (p.Arg406Trp) mutation of the microtubule-associated protein tau $(M A P T)$ gene.

$D C 2$

DC2 is a 76-year-old left-handed male retired professor of English literature. He presented with an 11-year history of progressive prosopagnosia and personality change with social disinhibition and obsessionality. No history of delusions, hallucinations, or other perceptual disturbance was noted, with no known family history of dementia. The general neurologic examination was normal. Serial neuropsychological assessments over a 6-year interval demonstrated a largely stable profile with prominent anomia and recognition memory dysfunction; DC2's general neuropsychological data at the time of the experimental assessment are summarized in Table 1. DC2 fulfilled current consensus criteria for a syndromic diagnosis of bvFTD [10].

Serial registered $T_{1}$-weighted volumetric brain MRI demonstrated progressive cerebral volume loss involving 
the anterior temporal and frontal lobes, more marked on the right (Figure 1). Genomic screening excluded a C9OR72 mutation.

\section{Healthy control subjects}

Four healthy right-handed male control subjects (mean age, 67 years; range, 58 to 72 years; see Table 1) matched to NT for age $\left(t_{5}=0.04 ; P>0.05\right)$ and with similar educational and social backgrounds but no history of neurologic or psychiatric illness also were assessed.

This study was approved by the local research ethics committee under Declaration of Helsinki guidelines. All subjects gave informed consent to participate, and provided consent to publish.

\section{Experimental assessment}

The experimental set-up is schematized in Figure 2. A paintbrush $(14.5 \times 1 \mathrm{~cm}, 1$ "25 was suspended by using a cross-clamp from a rod positioned between two tablemounted retort stands, such that the rod (and the attached paintbrush) could be rotated freely by manipulating a handle attached to one end. The subject was positioned with his dominant hand resting palm-down on the table between the retort stands, and the apparatus was adjusted so that the paintbrush lightly tracked across the skin of the hand when the handle was rotated by the subject, using his nondominant hand. During the experiment, the paintbrush was randomly moved along the suspending rod from trial to trial, such that the brush either would contact the subject's hand ("self" condition) or would not contact the subject's hand ("non-self" trials); on "other" trials, the experimenter delivered the tactile stimulus by using an identical paintbrush, either in time with the subject's own action (synchronous condition) or with a short delay (around 1 second; asynchronous condition). The retortmounted paintbrush was shifted by the experimenter before every trial (whether self or non-self) to minimize any extraneous cues from sound or the absolute position of the brush. The subject was blindfolded and instructed to rotate the handle 3 times in every trial: the task on each trial was to decide whether the brush stimulus was generated by the subject's own action or by that of the experimenter. It was established before commencing the experiment that subjects were able reliably to detect the sensory stimulus delivered by the brush. Thirty experimental trials were administered, comprising 10 self, 10 non-self synchronous, and 10 non-self asynchronous trials in randomized order. Subject responses were recorded and stored for offline analysis. No time limit was imposed, and no feedback about performance was given during the test.

Patients' performance was compared with that of healthy control subjects by using the Crawford and Howell modified one-tailed $t$ test for significant differences in single-case studies [11] and with a significance threshold of $P<0.05$.

\section{Results}

Results on the experimental behavioral test are summarized in Table 1. Compared with healthy controls, NT exhibited a significant deficit of self-other differentiation over all experimental conditions $\left(t_{5}=-3.48 ; P<0.021\right)$. Analyses of the three experimental conditions separately revealed that NT's performance was flawless for attribution of self-generated actions, whereas he performed significantly worse than healthy controls for attribution of both synchronous, non-self-generated actions $\left(t_{5}=-4.05\right.$; $P<0.008)$ and asynchronous, non-self-generated actions $\left(t_{5}=-5.5, P<0.001\right)$. NT's errors were therefore entirely overattributions of the experimenter's actions on non-self trials as self-generated responses. Like NT, patients DC1 and $\mathrm{DC} 2$ correctly attributed all self-generated actions. However, in contrast to NT, DC1 and DC2 showed no significant differences in performance relative to healthy controls on either of the non-self conditions (DC1: overall $t_{5}=0.12$, synchronous $t_{5}=0.45$, asynchronous $t_{5}=$ -0.78; DC2: overall $t_{5}=-0.36$, synchronous $t_{5}=-1.34$,
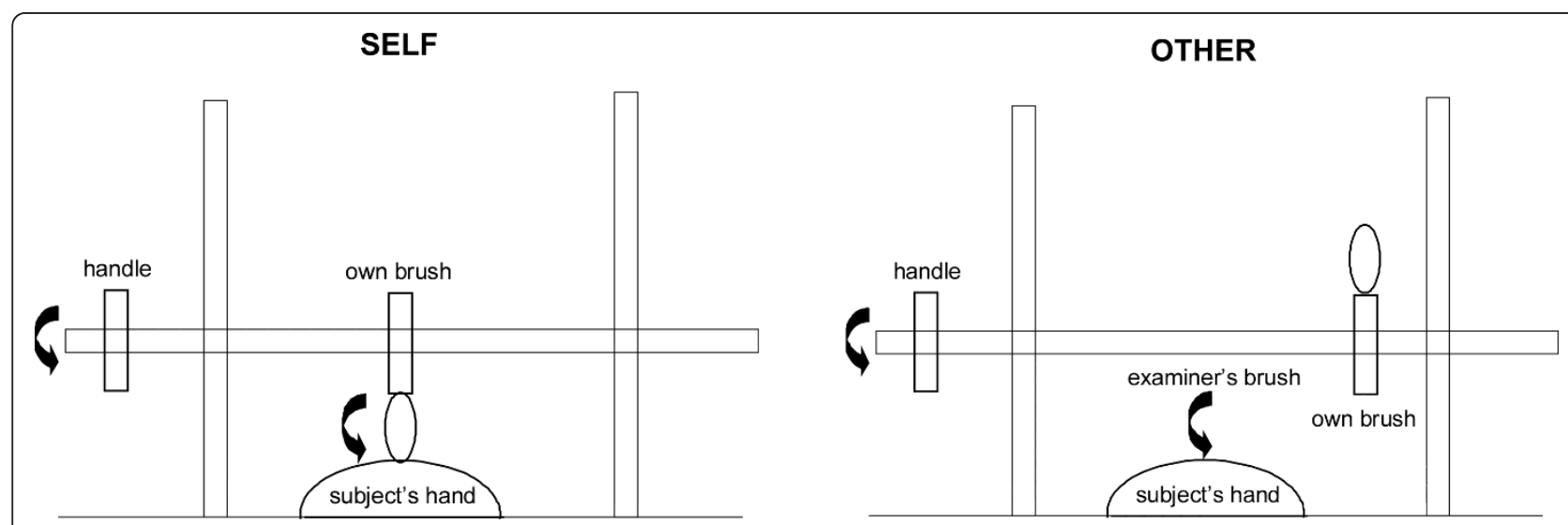

Figure 2 Schematic diagram of the experimental setup in the "self" and "other" action task conditions. See text for further explanation. 
asynchronous $t_{5}=0.78$; all $\left.P>0.05\right)$, and each patient's performance fell within the control range for every condition.

\section{Discussion}

It is evident from the range of scores (Table 1) that healthy controls and patients alike performed generally rather poorly on the synchronous non-self condition; performance differences were exposed largely in the asynchronous condition. This pattern of results suggests that the experimenter was able to control accurately the degree to which the external stimulus simulated (or did not simulate) the effect of a self-generated action.

\section{Conclusions}

Here we have shown that differentiation of the effects of one's own versus others' actions may become impaired in frontotemporal dementia associated with the C9ORF72 expansion. Indeed, the deficit of self-other differentiation appeared disproportionate to NT's otherwise relatively mild cognitive phenotype. As this patient's cognitive evolution was otherwise so indolent, we hypothesize that impaired self-other differentiation may be a key feature in the development of the complex behavioral disturbances that accompany the C9ORF72 expansion, or may to lead the development of moretypical cognitive deficits. The specificity of this finding for C9ORF72-associated frontotemporal dementia remains to be resolved. However, the present data suggest that the ability to distinguish one's own from others' actions is not comparably affected in other forms of frontotemporal dementia (including $M A P T$-associated disease); nor does it appear to be simply a consequence of more severe disease, as DC1 and DC2 were both substantially more cognitively impaired than was NT. Together, these findings raise the possibility that impaired self-other differentiation is a behavioral signal of C9ORF72 mutations. It is of interest that NT tended to "overattribute" others' actions to his own agency. A similar overattribution bias to self for actions of ambiguous origin has been reported both in healthy individuals $[12,13]$ and in schizophrenia [14]. Both healthy controls and the two patients without the C9ORF72 expansion here made self-overattribution errors in the synchronous non-self condition, but (unlike NT) were able to use the increased temporal delay in the non-self asynchronous condition to identify the external origin of the action. Although the overattribution to self of external actions may seem somewhat paradoxic in a condition such as schizophrenia with delusions of external control, it has been argued [6] that such a deficit might lead to an impaired ability to model one's own versus others' actions and an abnormal sense of invasion by external forces masquerading as oneself.
Previous theoretic accounts of the neurobiologic basis for sense of agency $[9,15]$ have accorded the cerebellum a key role as a comparator of efferent and afferent motor commands. However, the interpretation of agency is likely to engage a distributed brain network also including thalamus and posterior parietal cortex for transmission and integration of the sensory consequences of actions and prefrontal and cingulate cortex for cognitive appraisal of integrated percepts [16]. Impaired ability to distinguish the effects of one's own from others' actions might therefore potentially result from impaired prediction coding in the cerebellum or defective integration of sensory percepts by the thalamus or the parietal or prefrontal cortex $[5,7,17]$. The elements of this distributed network have been implicated in neuroimaging $[1,18]$ and neuropathologic $[4,18,19]$ studies of patients with C9ORF72 expansions. Although neuroanatomic correlation was not possible here, we hypothesize that dysfunction of the previously delineated cortico-thalamo-cerebellar network may have underpinned the behavioral deficit of self-other differentiation exhibited by NT $[5,7,9]$. Although impaired self-other action attribution has not, to our knowledge, been proposed previously as a general mechanism of behavioral decline in frontotemporal dementia, complex behavioral phenotypes remain poorly characterized in pathophysiologic terms. It may be that a range of more basic deficits is expressed in broadly similar behavioral phenotypes, within which certain features (for example, early prominent neuropsychiatric symptoms) may give a more-specific clue to the key pathophysiologic mechanism at work with particular mutations. We propose that impaired self-other differentiation in patients with C9ORF72 mutations may index a generic mechanism of defective own-action modeling and representation that may be somewhat analogous to the deficit proposed previously in patients with schizophrenia (6). Such a pathophysiologic mechanism could potentially be expressed in a range of clinical neuropsychiatric phenomena.

We present this single-case analysis with a number of caveats and suggestions for future work. Foremost among these, the findings require replication in a cohort of patients with the C9ORF72 expansion, with both clinical and neuroanatomic correlation. This patient did not exhibit clinically overt psychotic symptoms (delusions, hallucinations, or other perceptual disturbances); it would be particularly pertinent to test our hypothesis in a group of patients who do exhibit such symptoms (for example, delusions). The proposed role of the putative cortico-thalamocerebellar network could be directly assessed in a structural or functional brain-imaging paradigm. The use of other tasks designed to explore self-other differentiation in this population would further corroborate the present findings. The specificity of the deficit for C9ORF72-associated frontotemporal dementia requires further corroboration 
through comparison with larger cohorts of patients representing other forms of genetic and sporadic frontotemporal dementia. In addition, within the C9ORF72 expansion group, a need exists to establish the time course of development of the deficit in a longitudinal analysis. If our conjecture is correct, impaired self-other differentiation might manifest as an early feature, possibly even presymptomatically. The paradigm we propose is relatively straightforward and could be adapted to group longitudinal applications. We hope that our preliminary observation stimulates further hypothesis-led work directed to establishing the pathophysiologic basis of the complex behavioral disorders that characterize this newly discovered entity.

\section{Abbreviations}

BPVS: British Picture Vocabulary Scale; bvFTD: behavioral variant frontotemporal dementia; CBI: Cambridge Behavioural Inventory; D-KEFS: Delis-Kaplan Executive Function System; DS: digit span; GNT: Graded Naming Test; MRI: magnetic resonance imaging; NART: National Adult Reading Test; PIQ: Performance Intelligence Quotient; RMT: Recognition Memory Test; VIQ: Verbal Intelligence Quotient; VOSP: Visual Object and Space Perception; WASI: Wechsler Abbreviated Scale of Intelligence.

\section{Acknowledgements}

We are grateful to all the subjects for their participation. This work was undertaken at $\mathrm{UCLH} / \mathrm{UCL}$, who received a proportion of funding from the Department of Health NIHR Biomedical Research Centres funding scheme. The Dementia Research Centre is an Alzheimer's Research UK Co-ordinating Centre. This work was funded by the Wellcome Trust and by the UK Medical Research Council. SJC is supported by an Alzheimer's Research UK Senior Research Fellowship. JDW is supported by a Wellcome Trust Senior Clinical Fellowship (grant 091673/Z/10/Z).

\section{Authors' contributions}

All authors read and approved the manuscript. LED was involved in study planning, design, and coordination, acquired and analyzed the behavioral data, and was involved in drafting and critically revising the article. CJM was involved in study design, acquisition of neuroimaging data, and drafting and critically revising the article. MNR assessed the patients clinically and was involved in study design and in drafting the article. SJC and JDW obtained funding for and supervised the study, and were involved in study planning and design, in data collection, and in drafting and critically revising the article.

\section{Competing interests}

We have no financial competing interests to declare. LED, CJM, SJC, and JDW receive salary and research support from the Medical Research Council, Alzheimer Research UK, and the Wellcome Trust. MNR sits on the Data Monitoring Committee for Servier DMC Phase 2B AD Study 538093, and also sits on the Bapineuzumab Independent Safety Monitoring Committee for Janssen Al/Pfizer.

Received: 2 April 2012 Revised: 3 June 2012 Accepted: 13 August 2012 Published: 13 August 2012

\section{References}

1. Mahoney CJ, Beck J, Rohrer JD, Lashley T, Mok K, Shakespeare T, Yeatman T, Warrington EK, Schott JM, Fox NC, Hardy J, Colling J, Ravesz T, Mean S, Warren JD: Frontotemporal dementia with the C9ORF72 hexanucleotide repeat expansion: clinical, neuroanatomical and neuropathological features. Brain 2012, 135:736-750.

2. Hodges J: Familial frontotemporal dementia and amyotrophic lateral sclerosis associated with the C9ORF72 hexanucleotide repeat. Brain 2012, 135:652-655.

3. Snowden JS, Rollinson S, Thompson JC, Harris JM, Stopford CL, Richardson AM, Jones M, Gerhard A, Davidson YS, Robinson A, Gibbons L,
Hu Q, DuPlesses D, Neary D, Mann DM, Pickering-Brown SM: Distinct clinical and pathological characteristics of frontotemporal dementia associated with C9ORF72 mutations. Brain 2012, 135:693-708.

4. Arighi A, Fumagalli GG, Jacini F, Fenoglio C, Ghezzi L, Pietroboni AM, De Riz M, Serpente M, Ridolfi E, Bonsi R, Bresolin N, Scarpini E, Galimberti D: Early onset behavioral variant frontotemporal dementia due to the C9ORF72 hexanucleotide repeat expansion: psychiatric clinical presentations. J Alzheimers Dis 2012, 31:447-452.

5. Blakemore SJ, Wolpert D, Frith C: Why can't you tickle yourself? Neuroreport 2000, 11:R11-R16.

6. Franck N, Farrer C, Georgieff N, Marie-Cardine M, Dalery J, d'Amato T, Jeannerod M: Defective recognition of one's own actions in patients with schizophrenia. Am J Psychiatry 2001, 158:454-459.

7. Schmahmann JD, Weilburg JB, Sherman JC: The neuropsychiatry of the cerebellum: insights from the clinic. Cerebellum 2007, 6:254-267.

8. Schmahmann JD, Pandya DN: Disconnection syndromes of basal ganglia, thalamus, and cerebrocerebellar systems. Cortex 2008, 44:1037-1066.

9. Frith CD, Blakemore SJ, Wolpert DM: Abnormalities in the awareness and control of action. Phil Trans R Soc Lond B Biol Sci 2000, 355:1771-1788.

10. Rascovsky K, Hodges JR, Knopman D, Mendez MF, Kramer JH, Neuhaus J, van Swieten JC, Seelaar H, Dopper EG, Onyike CU, Hillis AE, Josephs KA, Boeve BF, Kertesz A, Seeley WW, Rankin KP, Johnson JK, Gorno-Tempini ML, Rosen H, Prioleau-Latham CE, Lee A, Kipps CM, Lillo P, Piguet O, Rohrer JD, Rossor MN, Warren JD, Fox NC, Galasko D, Salmon DP, Black SE, Mesulam M, Weintraub S, Dickerson BC, Diehl-Schmid J, Pasquier F, Deramecourt V, Lebert F, Pijnenburg Y, Chow TW, Manes F, Grafman J, Cappa SF, Freedman M, Grossman M, Miller BL: Sensitivity of revised diagnostic criteria for the behavioural variant of frontotemporal dementia. Brain 2011, 134:2456-2477.

11. Crawford JR, Howell DC, Garthwaite PH: Payne and Jones revisited: estimating the abnormality of test score differences using a modified paired samples $t$ test. J Clin Exp Neuropsychol 1998, 20:898-905.

12. Daprati E, Franck N, Georgieff N, Proust J, Pacherie E, Dalery J, Jeannerod M: Looking for the agent: an investigation into consciousness of action and self-consciousness in schizophrenic patients. Cognition 1997, 65:71-86.

13. van den Bos $E$, Jeannerod M: Sense of body and sense of action both contribute to self-recognition. Cognition 2002, 85:177-187.

14. Hauser M, Knoblich G, Repp BH, Lautenschlager M, Gallinat J, Heinz A, Voss M: Altered sense of agency in schizophrenia and the putative psychotic prodrome. Psychiatry Res 2011, 186:170-176

15. Frith CD: Consciousness, information processing and the brain. J Psychopharmacol (Oxford, England) 1992, 6:436-440.

16. Jeannerod M: The sense of agency and its disturbances in schizophrenia: a reappraisal. Exp Brain Res [Experimentelle Hirnforschung Experimentation cerebrale] 2009, 192:527-532.

17. Schmahmann JD, Caplan D: Cognition, emotion and the cerebellum. Brain 2006, 129:290-292.

18. Whitwell JL, Weigand SD, Boeve BF, Senjem ML, Gunter JL, DeJesusHernandez M, Rutherford NJ, Baker M, Knopman DS, Wszolek ZK, Parisi JE, Dickson DW, Petersen RC, Rademakers R, Jack CR jr, Josephs KA: Neuroimaging signatures of frontotemporal dementia genetics: C9ORF72, tau, progranulin and sporadics. Brain 2012, 135:794-806.

19. Al-Sarraj S, King A, Troakes C, Smith B, Maekawa S, Bodi I, Rogelj B, AlChalabi A, Hortobagyi T, Shaw CE: p62 positive, TDP-43 negative, neuronal cytoplasmic and intranuclear inclusions in the cerebellum and hippocampus define the pathology of C9orf72-linked FTLD and MND/ ALS. Acta Neuropathol 2011, 122:691-702.

doi:10.1186/alzrt145

Cite this article as: Downey et al.: Impaired self-other differentiation in frontotemporal dementia due to the C9ORF72 expansion. Alzheimer's Research \& Therapy 2012 4:42. 